\title{
Research on the System of Juvenile Criminal Responsibility Age in China Wei Gao
}

\author{
School of North China Electric Power University, Beijing 102206, China; \\ foggy1009vv@163.com
}

\begin{abstract}
With the development of economy and the progress of society, the physical, psychological and mental development of minors, compared with 1980s, in 1990s has been significantly faster. In recent years, Social concern for juvenile delinquency is rising, juvenile delinquency toward the age of low, the crime of adult, intelligent, criminal means cruel and violent direction. The factors closely related to ju venile delinquency is juvenile criminal responsibility and criminal responsibility age. At the same time, the age of criminal responsibility is not enough to achieve the purpose of preventing and punishing the crime of juvenile delinquency. In order to further study the actual situation of the minor crime in China, explore effective ways to prevent and punish juvenile delinquency, to determine the age and conditions of juvenile criminal responsibility is more reasonable, this paper explores and discusses the age system of juvenile criminal responsibility in China, and compared with other minor criminal responsibility age system, in order to analyze the drawbacks and limitations of the system of juvenile criminal responsibility age, and puts forward some suggestions and measures to solve these problems.
\end{abstract}

Keywords: Juveniles; Criminal responsibility age system; Foreign legal system; Legislative perfection suggestion

\section{我国未成年人的刑事责任年龄制度问题研究}

\author{
高薇 \\ (1.华北电力大学 人文与社会科学学院法学专业, 北京市 102206 )
}

摘要: 随着经济的发展与社会的进步, 未成年人的生理、心理年龄以及智力发育与二十世纪八、九十年代相比已经明显 增快, 近些年来, 社会上对于未成年人犯罪的关注度不断上升, 未成年人犯罪向着年龄趋低化、犯罪方式成人化、智能化、 犯罪手段残忍且暴力的方向发展, 而与未成年人犯罪密切相关的因素, 是未成年人的刑事责任以及其刑事责任年龄, 同时期 刑事责任年龄的规定已不足以达到预防和惩戒现在社会未成年人犯罪的目的。为了进一步研究在中国目前未成年人触犯刑法 的实际状况，探索对于防患和惩戒治理未成年人犯罪的有效途径，使确定未成年人承担刑事责任的年龄和条件更加合理，本 文探究和讨论了我国的未成年人刑事责任年龄制度, 并且与其他未成年人刑事责任年龄制度进行了比较, 以此来分析我国未 成年人刑事责任年龄制度仍然存在的弊端与限制, 并针对这些弊端和不足提出改进的建议和措施。

关键词: 未成年人；刑事责任年龄制度；国外法律制度；立法完善建议

中图分类号：DF613 文献标志码：B

\section{1 刑事责任年龄制度以及我国未成年人刑事责任年龄制度的现状}

\section{1 刑事责任年龄制度}

按照普遍的立法状况来看，一般所说的刑事责任年龄，是实施犯罪的人对于其触犯法律的行为应当承 担刑事责任所应该到达的年龄, 联系立法国家青少年的实际成长状况以及预防、惩治犯罪的现实需要, 现 代世界各国一般把刑事责任年龄划分为几个阶段，这一划分的依据一般是刑事责任年龄的逐步发展与年龄 因素、各个国家自身青少年儿童实际情况以及国家打击犯罪的实际需要。但不同的法系或者国家在划分的 方法上不尽相同，主要类型有两分制、三分制和四分制。选择两分制的国家，将刑事责任年龄进行了两个 
阶段的划分, 分为了绝对无责任年龄、完全负责任年龄, 根据 1954 年《格陵兰刑法典》: “凡不满 15 岁 的儿童实施的行为，不适用本法典。”这是格陵兰国家对于刑事责任年龄制度的立法规定。采取四分制的 国家，对于刑事责任年龄进行了四个阶段的划分：绝对不承担刑事责任年龄阶段、相对承担刑事责任年龄 阶段、减轻刑事责任年龄阶段、完全承担刑事责任年龄阶段。采取三分制的国家，对刑事责任年龄的划分 为: 完全不负责任年龄阶段、相对负责任年龄阶段、完全责任年龄阶段这三个阶段，比如中国。在当前大 多数国家的刑法规定中，一般都采取三分制或四分制的刑事责任年龄规定。

\section{2 我国未成年人刑事责任年龄法律制度的现状}

结合政治、经济、文化教育等方面的国家实际状况，结合青少年的成长特性，联系各种犯罪在中国的 现实情况，中国的刑法采用了 “教育为主、惩罚为辅” 的立法指导方针，在一定程度上借鉴了国外的有建 设性的法律，采用了三分制的方法，把刑事责任年龄按照三个不同的阶段进行了划分，即不满 14 周岁的 青少年对于其犯罪行为完全不承担刑事责任，已经满 14 周岁、仍未到 16 周岁的青少年负法律规定的相应 的刑事责任, 已经满 16 周岁的人对自己的犯罪行为要负全部的刑事责任, 这是我国刑法在第 17 条当中进 行了明确规定的三个阶段。

我国刑事责任年龄的划分的依据是犯罪人的犯罪行为能力、意志力、身心的成熟度，这其中的每个阶 段都符合相应年龄的生理以及心理成熟度，在完全刑事责任年龄阶段，已满 16 周岁的人生理和心理已经 达到了能够对于自己行为的性质、行为可能产生的后果具有全面的认识的成熟程度, 应当对于自己的刑事 犯罪负完全刑事责任; 已经达到 14 周岁但尚未达到 16 周岁的未成年人，也就是应当对自己触犯刑法的行 为承担相对刑事责任的年龄段的人, 已经拥有相当的行为能力和意志力, 可以对自身的行为作出一定程度 的认识和掌控，因此，对于我国刑法规定的八种有严重社会危害的犯罪类型而言，这类群体已经具有了一 定的认识和观念，可以意识到其所为的是严重、暴力犯罪，应当承担刑事责任。对于尚未满 14 周岁的未 成年人来说, 其因为生理和心理发育尚且不足, 不能够对于自己的行为性质以及行为所造成的后果产生法 律上的认识, 不具有对自己的行为负担刑事责任的能力, 因此将其划分为不承担刑事责任年龄阶段, 但对 于这个年龄的人, 其家长或监护人必须要加强管教, 因为刑法的划分虽然为其免除了刑事责任, 但不能完 全起到阻止犯罪的作用。

\section{2 不同法系未成年人刑事责任年龄的相关制度规定及分析}

\section{1 英美法系刑事责任年龄规定}

在未成年人刑事责任年龄的制度上，美国并没有形成统一、明确的规定，比如，在奥克拉荷马州，对 7 岁以下的儿童要求不承担刑事责任, 一些州要求最低年龄为 8 岁, 如内华达州、华盛顿, 规定应当负刑 事责任的最低年龄是 10 岁的是科罗拉多州, 将年龄界限划定为 12 岁有俄勒冈州等州, 美国的各个州在这 个制度上规定的最高的年龄是 13 周岁，例如佐治亚州、伊利诺斯州、纽约州等州，可见美国各州对于未 成年人刑事责任年龄的规定有非常大的差异。究其原因, 是美国制定并发展了发达的区际冲突法, 不同的 州在规定刑事责任年龄制度时, 根据其各自的实际情况作出不同的价值判断和选择。但非常明显的是, 我 们可以看, 对刑事责任年龄的下限所做出的界定, 美国各州普遍较低, 这也是因为美国社会正变的越来越 严重和低龄化的暴力犯罪形势所造成的。

\footnotetext{
1 参见郭绵庆. 熊伟伟《我国刑事责任年龄制度的缺陷与完善》[J]. 中国法院网, 2014（1）
} 
英国的相关立法规定中，对于刑事责任年龄方面，英国法律将其界定为三个阶段。绝对不负刑事责任 年龄阶段, 即没有实施犯罪行为的能力, 此阶段的年龄上限为十岁, 即未满十岁的儿童属于绝对不负刑事 责任年龄的阶段; 对于 14 岁以下、10 岁以上的儿童, 英国法律推定其没有进行犯罪的能力, 但是这种推 定不是绝对的，如果有证据对其反驳，其对于被证明的犯罪行为也应当承担相应的刑事责任; 14 岁以上的 人，是应当负刑事责任的。

通过上述国家的立法, 我们可以看出, 在英美法系国家的立法中, 虽然未成年人刑事责任年龄的规定 虽然不完全相同，但有一个共同的特点，即对于刑事责任年龄下限的规定使普遍较低的，这与英美等国家 资本主义经济制度、资产阶级政治制度已经文化背景是密切相关的。

\section{2 大陆法系刑事责任年龄规定}

在大陆法系国家, 以意大利为例, 以具有正常的理解能力和意思表示能力为标准, 规定不满 14 周岁 的人无法达到一定的成熟标准，也不具有健康的心理素质和成熟的心理意识，因此不满 14 周岁的人的犯 罪行为将被推定为无罪行为。另一个大陆法系国家的例子是俄罗斯, 俄罗斯刑法的概念当中，未成年人是 指 “当他犯罪时已经达到 14 周岁、但仍小于 18 周岁的人”，没有达到 14 周岁则被规定为幼年 。同时， 对于从 14 岁开始承担刑事责任的人，规定其担责的罪名范围明显缩小，严格限制在几种严重的刑事犯罪 中。

同样作为大陆法系国家的我国，对于刑事责任年龄的规定与其他大陆法系国家存在着较高的相似度， 与英美法系相比, 规定的刑事责任年龄下限的较高, 并且在相对承担刑事责任年龄的阶段, 我国同样规定 了必须承担刑事责任的几种严重危及社会和公共财产以及安全的罪行。

\section{3 对于我国未成年人刑事责任年龄制度的缺陷分析及完善建议}

\section{1 我国刑事责任年龄制度的缺陷分析}

\section{1.1 存在的缺陷}

我国当初进行《刑法》的制定之时，对于刑事责任年龄制度的相关规定，其主要目的是 “惩罚为主、 预防为辅” , 从而最大限度的保护未成年人的健康成长。然而三十多年过去了, 未成年人犯罪屡见不鲜, 且犯罪率逐年上升, 甚至有未成年人以法律作为挡箭牌实施犯罪，对他人的生命财产安全造成威胁，这些 现象对我国的社会公共安全造成了极大的危害，结合我国未成年人犯罪的趋势，也就是逐渐低龄化、成人 化、智能化、手段残忍暴力的趋势，又由于未成年人刑事责任、未成年人刑事责任年龄是与未成年人犯罪 密不可分的重要因素, 同时期刑事能力责任年龄的规定已不足以达到预防和惩戒现在社会未成年人犯罪的 目的。并且在未成年人相对承担刑事责任年龄阶段的规定中， 《中华人民共和国刑法》的表述模糊不清， 学界主要存在 “罪名说” 以及 “犯罪行为说” 两种分歧。

\section{1 .2 成因分析}

现代社会人的身体、心智发育比三十年前提前了至少 2-3 年, 但是我国刑法在这一方面的相关规定也 早已实施了二十多年, 立法时据以考虑的各种客观因素早已产生巨大变化, 尤其是经济环境。伴随着信息 网络时代的到来以及媒体的迅速发展, 未成年人接触到的信息与日俱增, 且良莠不齐, 其中包含了大量的 色情、暴力的不良因素, 但也包含了大量的法制、法治理念, 尤其是教育的普及范围的扩大和教育质量的 
提升，使未成年人接受道德法律信息和道德信息已经达到了与之前不可同日而语的水平。 ${ }^{2}$

\section{2 我国刑事责任年龄制度的完善建议}

\section{2.1 加强司法实践中对于年龄认定的监督}

对未成年人年龄证据形式上的瑕疪进一步严格规定，对证据实质内容上的错误建立纠正制度，加大对 于当事人和司法人员的监督和惩罚力度, 防止当事人以及相关办案人员弄虚作假, 使应当受到惩罚的犯罪 行为逃脱法律的制裁。

\subsection{2 明确相对负刑事责任年龄的范围}

在我国刑法关于相对承担刑事责任阶段的规定中，应当承担刑事责任的严重危害社会的八类犯罪，应 该界定为 “犯罪行为” 而非 “罪名” 。我国在 1997 年修订刑法时, 调查和实证分析工作均存在不足, 立 法技术尚不成熟, 修订较为仓促, 需要进行进一步的完善和修改。在未成年人暴力犯罪日趋低龄化、成熟 化、智能化、团伙化的大环境之下，若只是将这八种犯罪理解为 “罪名” 而不是 “犯罪行为” ，加之我国 严格适用 “罪刑法定” 原则, 会造成危害性相当、性质相似的犯罪只能部分定罪处罚的结果, 造成罪刑不 均衡。

\section{2 .3 适当降低完全不负刑事责任年龄的下限}

结合我国当前的社会现实, 由未成年人实施的犯罪行为的数量是在迅速增长的, 犯罪现状十分严重, 犯罪的年龄也在日益降低, 建议把未成年人完全不负刑事责任的最低年龄下降至十三周岁。由于现代社会 物质文化水平的提高, 当代未成年人心理、生理发育较快, 十三周岁已经完成了基本的小学教育, 对于社 会已经能够形成自己独立的主观认识, 这一方面能够提高未成年人的辨认能力和控制能力, 另一方面也会 在无形之中扩大未成年人犯罪的主观恶性, 未成年人十三周岁时已经能够基本理解自己行为的性质和意 义, 综合我国目前的社会经济状况以及未成年人犯罪等因素, 认为适当对未成年人刑事责任年龄下限进行 降低是具备合理性与可行性的。

\section{4 结语}

未成年人的成长与国家、社会的发展密切相关, 为了社会的和谐、稳定的发展, 为了未成年人能够健 康成长, 对于未成年人犯罪的预防和惩治显得越来越重要, 如何对未成年人犯罪嫌疑人进行教育和改造, 是法学界乃至全社会都紧密关注的问题。在本文中, 分析和探讨了未成年人犯罪的刑事责任年龄制度, 横 向比较了中国及域外的未成年人刑事责任年龄制度，论述了我们国家未成年人刑事责任年龄制度现存的一 些弊端和不足, 就有关缺陷提出了具有针对性的完善建议, 但由于写作水平和法学理论功底有限, 还存在 诸多不足, 但相信随着依法治国不断推进以及社会主义法治建设的不断完善, 我国的未成年人刑事责任年 龄制度一定会向着更加合理的方向发展。

\section{参考文献:}

[1]王作富, 黄京平. 《刑法》[M]. 北京:中国人民大学出版社, 2010 .

$[2]$ 韩玉风, 《从比较法学角度浅谈主要国家未成年人刑事责任年龄的异同》 $[\mathrm{J}]$. 天津市政法管理干部学院学报, 2009

[3]何家弘, 张卫平. 《外国证据法选择》 [M]. 北京: 人民法院出版社, 2000.

[4]党日红，《俄罗斯未成年人刑事责任年龄规制模式研究》 [J]. 中华女子学院学报, 2010.

[5]殷淑娟，《论我国未成年人刑事责任年龄制度的完善》[D]. 辽宁大学, 2015.

2 参见刘佳音. 《未成年人刑事责任年龄司法认定制度完善研究》[D].吉林大学. 2016. 
[6]姚建龙， 《少年刑法与刑法改革》 [M]．北京：中国人民公安大学出版社，2005.

[7]林维，《未成年人刑事责任年龄及其制裁的新理念》 [J]。国青年政治学院报， 2005.

\section{Reference}

[1] Wang Zuofu, Huang Jingping Criminal Law [M]. Beijing: Renmin University of China Press, 2010.

[2] Han Yufeng On the Similarities and Differences of Juvenile Criminal Responsibility Age in Major Countries from the Perspective of Comparative Law [J].Journal of Tianjin Administrative Cadre Institute of Politics and Law, 2009

[3] He Jiahong, Zhang Weiping Selection of Foreign Evidence Law [M]. Beijing: People's Court Press, 2000

[4] Dang Rihong A Study on the Age Regulation Model of Russian Juvenile Criminal Responsibility [J]. Journal of China Women's College, 2010

[5] Yin Shujuan Discussion on the Perfection of the Age System of Juvenile Criminal Responsibility in China [D]. Liaoning University, 2015.

[6] Yao Jianlong Juvenile Criminal Law and Criminal Law Reform [M]. Beijing: Chinese People's Public Security University Press, 2005.

[7] Linwei the New Concept of Juvenile Criminal Responsibility Age and Its Sanction [J]. Chinese Journal of Youth Politics, 2005. 